

\title{
RELACIÓN ENTRE TRASTORNO DE CONDUCTA ALIMENTARIA Y GÉNERO Y FAMILIA EN ADOLESCENTES ESCOLARIZADOS, SUBA (BOGOTA)
}

\author{
Relationship between Eating Disorders with Gender and Family in Adolescents \\ Attending Schools in Suba, Bogotá
}

\author{
Diana Carolina Martínez, Sonia Gisela Martínez. ${ }^{\ddagger \neq \neq}$ \\ Tutor: Juan Carlos González $\$ \S \S \S$ \\ Colaboración en el análisis estadístico y edición: Jenny Pinzón ${ }^{* * * * *}$ \\ Dibujos: Andrés Reyes ${ }^{++\dagger+}$
}

\begin{abstract}
Resumen
Objetivo: establecer si existe relación entre el riesgo de desarrollar un trastorno de conducta alimentaria (TCA) y el género y la estructura y funcionalidad de la familia.

Metodología: se aplicó una encuesta entre estudiantes que cursaban los grados sexto a once, en cuatro colegios de la localidad de Suba, indagando por variables de género, estructura y funcionalidad familiar (a través del test de APGAR familiar); se realizó el Test de Scoff para identificar la presencia de TCA.

Resultados: se encuestaron 3217 adolescentes, de los cuales 1603 eran hombres y 1614 mujeres. El $20 \%$ de los hombres presenta riesgo de TCA frente a un $30 \%$ de las mujeres. En relación a la estructura familiar de la población que presentó TCA, el $25,5 \%$ refirió tener familia nuclear incompleta, mientras que el 24,2 \% familia nuclear completa. Respecto a la funcionalidad familiar, el 33,6 \% percibió disfunción severa y el 22,9 \% buena función. En este estudio el género y la función familiar mostraron relación estadística con los TCA a diferencia de la estructura familiar.

Conclusiones: las mujeres tienen mayor predisposición a presentar un TCA. La disfunción familiar es un factor que influye en el desarrollo de un TCA.

Palabras clave: Trastornos de alimentación y de la ingestión de alimentos, adolescente, relaciones familiares, familia, riesgo.

Abstract

Objective: To establish the relationship between the risk of developing an eating disorder with gender and family (structure and functionality).

Methodology: A survey was conducted on Sixth to Eleven-Grades students in four schools located in Suba, a locality of Bogotá. The survey inquired about sociodemographic variables including family structure. Family functionality was looked into by the Apgar score and, eating disorders by the Scoff Score.

Results: 3197 adolescents were surveyed, of which 1603 were men and 1614 were women. Eating disorder risk was evaluated in $20 \%$ of male population and, in $30 \%$ of female one. Among who presented an eating disorder, 25,5\% reported having an incomplete nuclear family and, 24,2\% a nuclear family. Likewise, 33,6 \% perceives severe dysfunction family and, 22, $9 \%$ normal functionality. Gender and family functionality showed relation with eating disorders unlike family structure.
\end{abstract}

Conclusions: Women are more predisposed to present an eating disorder. On the other hand, family dysfunction is a factor that contributes to this disorder.

Keywords: Feeding and eating disorders, adolescent, family relations, family, risk.

$¥ \neq \ddagger \ddagger$ Estudiantes VII semestre - Facultad de Medicina FUJNC

$\S \S \S$ MD. Especialista Medicina Familiar Integral, MSP. Director Departamento de Medicina Comunitaria - juan.gonzalez@juanncorpas.edu.co ${ }^{* * * * *}$ Coeditora Carta Comunitaria - jenny.pinzon@juanncorpas.edu.co

${ }^{+++t+}$ MD Cirujano General 


\section{INTRODUCCIÓN}

Los trastornos de conducta alimentaria se definen como una percepción errónea de la imagen corporal y del peso $(1,2)$, asociada a una obsesión por la comida o al miedo a engordar, lo que resulta en problemas médicos y nutricionales $(3,4)$. Esta conducta es una respuesta a los impulsos psíquicos de la persona que se ve afectada (5), lo que hace pensar en una patología mental.

Es necesario tener en cuenta que el pronóstico no es bueno, por ejemplo, hay un $50 \%$ de personas con anorexia nerviosa que, a pesar de haber tenido un tratamiento hospitalario, recae al continuar con los síntomas psicosociales, por su parte, la bulimia presenta resistencia al tratamiento lo que dificulta la recuperación (6).

El pronóstico deficiente se da por condiciones tales como la personalidad inestable, un matrimonio disfuncional y un largo período de padecer la enfermedad. Al contrario, las personas que tienen una autoestima alta, que reconocen su patología y que logran alcanzar un mayor grado de autonomía tienen un mejor pronóstico (6).

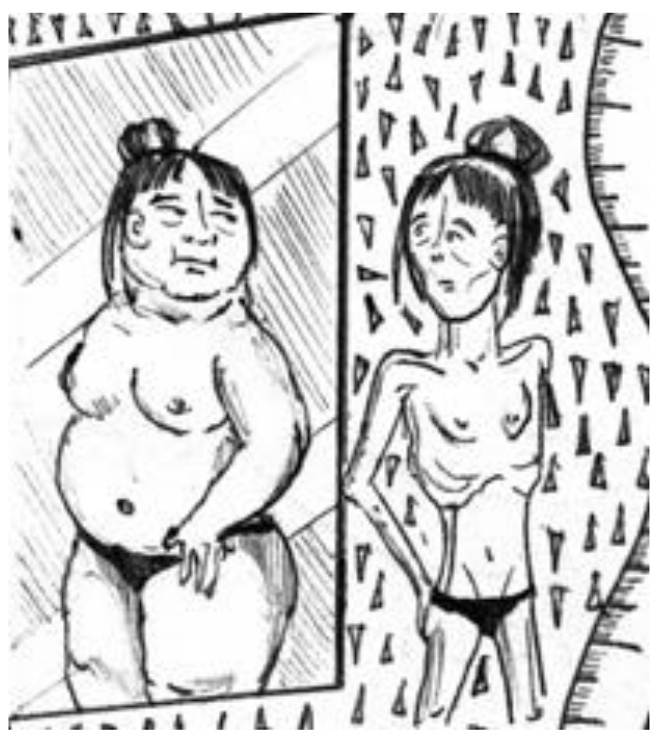

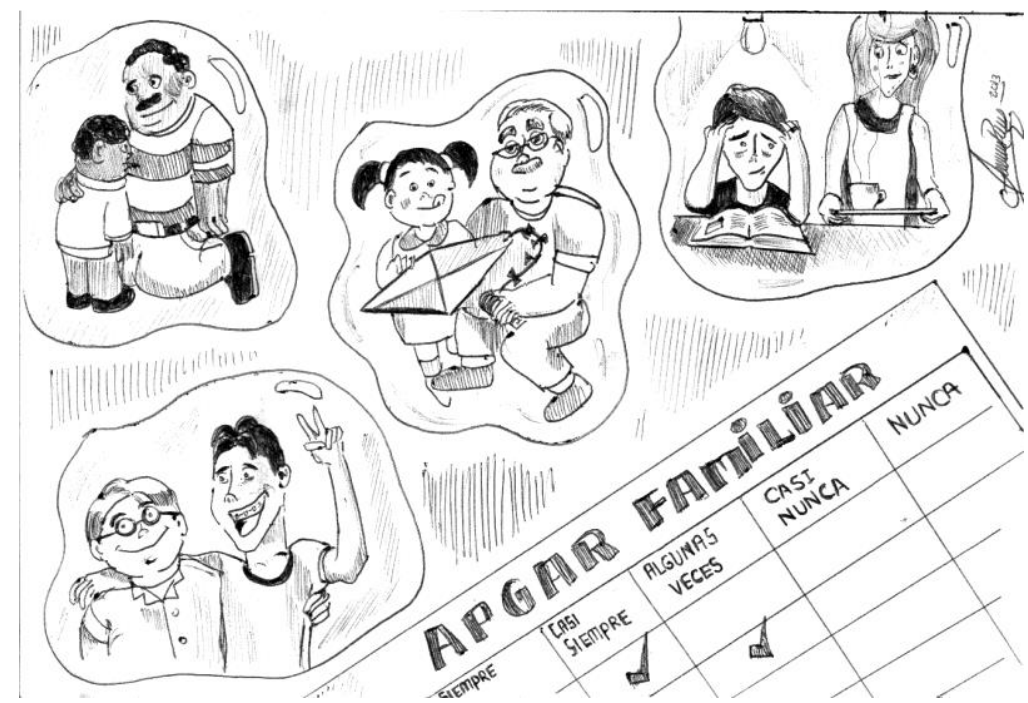

Se ha relacionado la disfunción familiar con el desarrollo, mantenimiento y permanencia de los trastornos alimentarios (4). Esta disfunción estaría dada por las dificultades que se presentan en los procesos de comunicación de la familia debido al aumento de la rigidez, la menor cohesión y la menor flexibilidad. En familias con estas características se puede ver un comportamiento desligado y caótico en comparación con las familias donde no hay TCA (7). La sobreprotección y la evasión de los problemas son también características del modelo de familia psicosomática en pacientes con TCA (8).
La disfunción familiar, que se suma a la baja autoestima, junto con el transmitir a los hijos la preocupación de conservar el peso y la figura, lo que refuerza de cierta manera la insatisfacción corporal, son aspectos en los que intervienen indirectamente las familias, los cuales se combinan aumentando la aparición de TCA $(8,9)$.

En cuanto a factores precipitantes de los TCA se han asociado actividades como la danza, el patinaje artístico y la gimnasia rítmica que motivan a las mujeres a adelgazar, en especial a las adolescentes, con el fin de conseguir un mejor rendimiento (10). 
Se ha demostrado que la incidencia de estos trastornos es mayor en las mujeres (8), debido a que su insatisfacción corporal es mayor que la de los hombres, aunque estos siguen teniendo un porcentaje significativo (11).



\section{MATERIALES Y MÉTODOS}

Tipo de estudio: Descriptivo de tipo corte transversal.

Población: Estudiantes que cursaban los grados sexto a once en los cuatro colegios evaluados en la localidad al momento de la encuesta.

Criterios de inclusión: estudiantes que pertenecieran a los grados sexto, séptimo, octavo, noveno, décimo y once de los colegios evaluados y que hubieran estado presentes el día y el momento de la encuesta, tanto en las jornadas mañana y tarde.

\section{Variables de estudio:}

Se indagó por aspectos sociodemográficos (edad, sexo, percepción del estrato). Se preguntó por la estructura del hogar (nuclear, completo e incompleto) y, por la función familiar, a través del Test de APGAR familiar (¿Me satisface la ayuda que recibo de $\mathrm{mi}$ familia cuando tengo algún problema o necesidad? ¿Me satisface cómo en mi familia hablamos y compartimos nuestros problemas? ¿Me satisface cómo mi familia acepta y apoya mi deseo de emprender nuevas actividades? ¿Me satisface cómo mi familia expresa afecto y responde a mis emociones tales como rabia, tristeza, amor? ¿Me satisface cómo compartimos en mi familia el tiempo para estar juntos, los espacios en la casa y el dinero?), con tres posibilidades de respuestas a cada una de estas preguntas (casi nunca $=0$ puntos, algunas veces $=1$ punto, y casi siempre $=2$ puntos). Se considera funcionalidad normal si se obtienen de 7 a 10 puntos; disfunción moderada de 4 a 6 puntos $y$, disfunción severa, de 0 a 3 puntos (14).

Se realizó el Test de Scoff (15) para evaluar el riesgo de TCA en los encuestados ( $¿ \mathrm{Te}$ hace sentir mal tener lleno el estómago? ¿Te preocupa haber perdido el control sobre cuánto comes? ¿Has perdido peso en los últimos tres meses?
Los medios de comunicación influyen en los estereotipos de belleza del adolescente. La televisión ejerce un bombardeo constante de imágenes que manipulan el pensamiento de los jóvenes poniendo en riesgo su salud mental (12). Se ha demostrado una estrecha relación entre la publicidad y los factores socioculturales para la creación del prospecto ideal de género e imagen corporal (13).

Lo anterior muestra que los factores que contribuyen al desarrollo de TCA están entrelazados. Este trabajo identifica el papel que cumplen la familia (su estructura y funcionalidad) y otros factores, como el género, en la prevalencia de los TCA en los adolescentes, siendo importante indagar en estos aspectos ya que dichos trastornos constituyen actualmente un problema de salud pública por el incremento de su incidencia $(4,13)$, la gravedad de sus síntomas y la resistencia al tratamiento que estos pacientes presentan (13).

Este artículo, al evidenciar los factores que influyen para que los adolescentes desarrollen un TCA, pretende contribuir para que, en un futuro, se logre una buena prevención así como la curación de los jóvenes que presentan este problema (4).

Se considera respuesta positiva una pérdida de peso en torno a los 6 kilogramos. ¿Te ves obeso a pesar de que los demás te dicen que estas demasiado delgado? ¿Dirías que la comida domina tu vida?). Si el test arrojaba dos respuestas positivas indicaba la posibilidad de TCA.

Prueba piloto: La encuesta utilizada para el presente artículo, ya ha sido probada y evaluada en otros estudios anteriores $(16,17)$.

Recolección de la información: inicialmente se obtuvo el permiso de las directivas de cada institución encuestada con el compromiso de obtener un diagnóstico que permita intervenir en la población. Posterior a esto, se ingresó a cada aula de clase explicando la finalidad de la encuesta, así como la libertad para contestarla y, sobre todo, la confidencialidad de los datos. Al final, cada encuestador recopiló la información obtenida en una base de datos y luego se consolidó todo en una sola tabla.

Manejo estadístico: se tabularon los resultados y se obtuvieron las frecuencias en el programa Excel. No todas las encuestas fueron respondidas en su totalidad, por ende, de cada pregunta se obtuvo el porcentaje del total contestado. Se unieron las bases de datos de los cuatro colegios estudiados en el período 2015-2016 con el fin de observar los riesgos psicosociales a los que está expuesta esta población.

A través de la escala del test de Scoff, se obtuvo la prevalencia de riesgo de TCA y, usando el programa Epi Info, se obtuvieron los Odds Ratio entre esta variable y el género, la función y la estructura familiar, respectivamente, así como los intervalos de confianza y la prueba $\mathrm{P}$ para determinar si había o no relación entre las variables comparadas. 


\section{RESULTADOS}

Se encuestó un total de 3217, de estos, 3197 tienen una edad que oscila entre los 10 y los 20 años.

Tabla 1. Características generales de la población encuestada en los cuatro colegios de la localidad de Suba.

\begin{tabular}{lrc}
\hline \multicolumn{1}{c}{ VARIABLE } & $\mathrm{N}^{\circ}$ & $\%$ \\
\hline Edades & 710 & 22 \\
De 10 a 12 años & 2155 & 67 \\
De 13 a 16 años & 332 & 10 \\
De 17 a 20 años & & \\
Sexo & 1603 & 50 \\
Masculino & 1614 & 50 \\
Femenino & & \\
Estrato & 1487 & 49 \\
Estratos 1 y 2 & 1505 & 50 \\
Estrato 3 y 4 & 38 & 1 \\
Estratos 5 y 6 & $N^{\circ}$ & $\%$ \\
Funcionalidad Familiar & 147 & 5 \\
Disfunción severa & 424 & 14 \\
Disfunción moderada & 2488 & 81 \\
Funcionalidad normal & \multicolumn{2}{c}{} \\
Estructura Familiar & 1649 & 53 \\
Hogar Nuclear (vive con papá, mamá y hermanos) & 1007 & 32 \\
\hline Hogar incompleto (falta uno de los padres) &
\end{tabular}

Tabla 2. Características de los adolescentes encuestados en torno a TCA.

\begin{tabular}{lcc}
\hline \multicolumn{1}{c}{ RIESGOS DE TRASTORNO DE CONDUCTA ALIMENTARIA } & N & \% \\
\hline Riesgo de trastorno de conducta alimentaria. & 798 & 25 \\
Se provoca el vómito después de comer. & 266 & 9 \\
Le preocupa haber perdido el control en su forma de comer. & 998 & 34 \\
Ha perdido más de 7 kilos en los últimos tres meses. & 407 & 14 \\
Cree que está gordo(a) así los demás le digan que esta delgado(a). & 590 & 20 \\
\hline
\end{tabular}

La tabla 2 muestra las preguntas realizadas a los adolescentes encuestados para valorar la presencia de TCA.

Tabla 3. Razones de riesgo para el desarrollo de TCA

\begin{tabular}{|c|c|c|c|c|c|c|c|c|}
\hline \multirow{3}{*}{ VARIABLE } & \multicolumn{4}{|c|}{ TCA } & \multirow{3}{*}{ OR } & \multirow{3}{*}{$\mathbf{L I}$} & \multirow{3}{*}{ LS } & \multirow{3}{*}{$\begin{array}{c}\text { Prueba } \\
p\end{array}$} \\
\hline & \multicolumn{2}{|c|}{ Sí } & \multicolumn{2}{|c|}{ No } & & & & \\
\hline & $\mathbf{N}^{\circ}$ & $\%$ & $\mathbf{N}^{\circ}$ & $\%$ & & & & \\
\hline \multicolumn{9}{|l|}{ Género } \\
\hline Hombre & 313 & 20,0 & 1252 & 80,0 & \multirow{2}{*}{0,6} & \multirow{2}{*}{0,5} & \multirow{2}{*}{0,7} & \multirow{2}{*}{0,000000} \\
\hline Mujer & 481 & 30,0 & 1101 & 69,6 & & & & \\
\hline \multicolumn{9}{|l|}{ Estructura familiar } \\
\hline Nuclear incompleta & 251 & 25,5 & 734 & 74,5 & \multirow{2}{*}{1,1} & \multirow{2}{*}{0,9} & \multirow{2}{*}{1,3} & \multirow{2}{*}{0,449283} \\
\hline Nuclear completa & 390 & 24,2 & 1224 & 75,8 & & & & \\
\hline \multicolumn{9}{|c|}{ Funcionalidad familiar } \\
\hline Disfunción severa & 49 & 33,6 & 97 & 66,4 & \multirow{2}{*}{1,7} & \multirow{2}{*}{1,2} & \multirow{2}{*}{2,4} & \multirow{2}{*}{0,003065} \\
\hline Buena función & 558 & 22,9 & 1882 & 77,1 & & & & \\
\hline
\end{tabular}

La tabla 3 evidencia que las variables relacionadas con el desarrollo de TCA en este estudio fueron el género y la funcionalidad familiar. La estructura familiar no mostró diferencia estadística respecto al riesgo de TCA. La variable más significativa fue género (prueba $p$ 0,000000). 


\section{DISCUSIÓN}

La presente investigación está sujeta a la limitación propia de las encuestas, es decir, que no se conteste con la verdad. Al respecto, se notaron unas pocas inconsistencias entre algunas respuestas; por ejemplo, en la pregunta de si ya habían recibido información acerca de planificación familiar contestaban en forma negativa, mientras que, en la siguiente pregunta (a qué edad recibió dicha información), escribían la edad. Sin embargo, tal limitación se enfrentó a través de la insistencia en la voluntariedad y el anonimato. La similitud obtenida en los resultados de cada colegio, hace suponer que se superó suficientemente como para darle validez. Además,

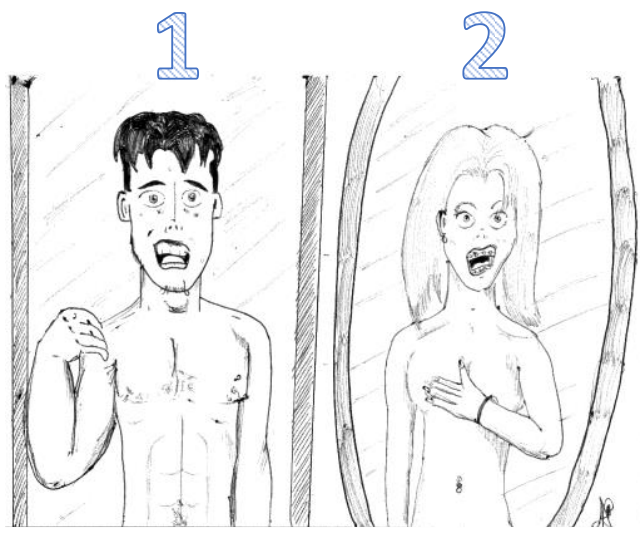

los resultados obtenidos en el presente trabajo guardan correlación con las referencias consultadas, por lo que se presume que este no fue un factor limitante a la hora de hallar veracidad en los resultados. Por otro lado, hay que tener en cuenta que para evaluar los TCA en la presente investigación, se utilizó el Test de Scoff en toda la población encuestada, prueba que hasta el momento solo ha sido validada en mujeres. Así que debemos someternos a un margen de error a la hora de detectar la presencia de TCA en la población masculina (18).

En este estudio se encontró una diferencia casi del doble (tabla 3, OR 0,6 IC95 \% 0,5-0,7) entre hombres y mujeres con TCA. Aunque no con la misma frecuencia, lo anterior concuerda con los resultados de las referencias revisadas $(19,20,21)$. Gracias a los medios de comunicación y al concepto de estética de nuestra sociedad, se ha transformado el concepto de la imagen corporal en un culto a la supuesta perfección, propia de una figura esbelta (22). En la población adolescente, esta idea llega a convertirse en una cuestión de decisión basada en un mundo glamoroso (22). Para lograrlo, las mujeres acuden al uso de laxantes, al vómito inducido y a la disminución en la ingestión de alimentos. Todo lo anterior, acompañado de la sensación de culpa por no lograr su objetivo (22). Esto podría ser una explicación de por qué las mujeres tienen una mayor prevalencia de TCA en las encuestas realizadas tanto en el presente trabajo como en las demás bibliografías acerca del tema.

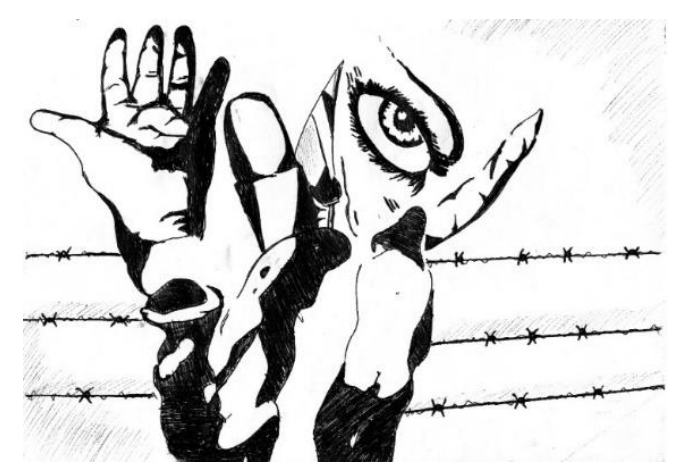

Otra explicación para un mayor riesgo en las mujeres de padecer de un TCA es que puede deberse a la presión que ejercen la sociedad y los medios de comunicación sobre el modelo ideal de belleza $(8,9,23,24)$, lo que hace a las jóvenes querer tener "un cuerpo socialmente deseable" (25). Todas estas ideas van ligadas a la autopercepción que la adolecente tenga. En la mayoría de los casos se presentan Ios TCA por la insatisfacción corporal $(4,23,26)$ y el deseo de adelgazar al sobreestimar el tamaño de su cuerpo (25, 26).

Las hipótesis acerca de por qué los hombres desarrollan TCA apuntan a culpar nuevamente a los medios de comunicación masiva, al enfatizar los ideales de fuerza física, fortaleza, agresividad, competitividad e independencia de los hombres (27). Han postulado también la idea de que el deporte desempeña un papel de vulnerabilidad en ellos por su exigencia (27).

Para las diferencias entre el origen de los trastornos en hombres y mujeres se ha postulado que mientras a las mujeres les interesa la delgadez, para los hombres es más importante el tener un cuerpo musculoso (20). Otra idea que se ha lanzado es que las mujeres tienden a sentirse insatisfechas con la parte inferior de sus cuerpos y los hombres con la parte superior (27). 


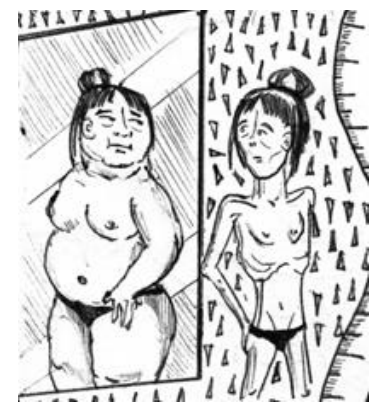

Sin embargo, no solo factores individuales sino también familiares influyen en la predisposición a desarrollar un TCA, así, "...al ser la familia el núcleo social más cercano al individuo y la responsable de la transmisión de valores, hábitos y costumbres...", (28) debemos considerarla como una influencia determinante para desarrollar un trastorno. La disfunción familiar es directamente proporcional al desarrollo de TCA; describiendo la falta de cohesión y la poca tolerancia al conflicto como determinantes para su desarrollo como este. Un factor relevante es el exceso de control por parte de los padres sobre los adolescentes, el ser sobreprotectores, autoritarios y el impedir su independencia (28), causando en los adolescentes la impresión de que no tienen el control sobre sus vidas, pensando así, que el único objeto sobre el que pueden ejercer o imponer sus decisiones es su cuerpo (28). Por otro lado, se ha demostrado que la falta de afecto y supervisión por parte de los padres puede resultar en

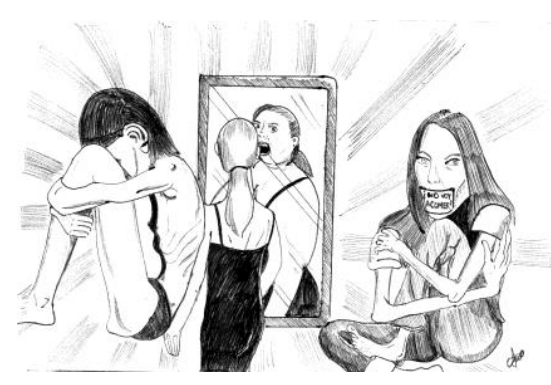

En dichas familias se perciben autoritarismo y roles estrictos en su relación, lo que sugiere rigidez dentro del entorno familiar (6). Por su parte la flexibilidad al verse afectada dificulta la interacción ante una crisis (24) sin dejar de lado la cohesión que limita la expresividad y saca a flote la carencia de apoyo y afecto que demandan los hijos.

Uno de los mayores problemas que se da es el evadir los conflictos (32), ya que se elimina la posibilidad que tienen para negociar sus diferencias, impidiendo así la solución a sus problemas (31). Estas situaciones llevan al caos y el desligamiento familiar (11) todo dado por la falta de comunicación (30). Hay que tener en cuenta que la rigidez también lleva a la evasión de los eventos que suceden en la familia (31). Como consecuencia de estos factores se impide al adolescente tener conciencia de su problemática (31).

Así queda claro que uno de los aspectos a evaluar es el papel que desempeña la familia para el desarrollo de un TCA, ya que podría ser una diana terapéutica. La funcionalidad familiar se puede evaluar con varias interacciones e, independientemente del autor, hay tres en común que son el aglutinamiento o cohesión, la rigidez y la resolución de conflictos. Estas tres son las bases de la relación familiar (7). adolescentes con baja autoestima, la cual guarda estrecha relación con el riesgo de desarrollar un TCA. Según estudios (28), la disfunción familiar es directamente proporcional al desarrollo de TCA y la falta de cohesión y la poca tolerancia al conflicto son determinantes para su desarrollo, apoyando así tanto lo dicho anteriormente como los resultados de la presente investigación.

Este estudio al igual que los documentos registrados anteriormente, evidencia el impacto que tiene la funcionalidad familiar, a diferencia de su estructura, sobre los TCA (tabla 3) $(29,30)$. Varios autores sustentan estos resultados teniendo como base las variables con que se evalúa la familia. La mayoría coinciden en un aumento de la rigidez, una disminución en la flexibilidad y la negación de los problemas $(11,31)$.

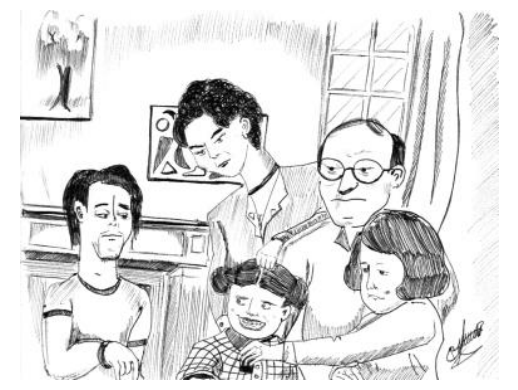

Se puede concluir que los trastornos de conducta alimentaria no han sido intervenidos adecuadamente, ya que al aumentar su incidencia se han convertido en un problema de salud en muchos países $(4,12,26)$. En esta investigación se analizaron factores que intervienen en la aparición de los TCA en los adolescentes con la intención de contribuir a la disminución de su prevalencia al usarlo como base para construir futuras dianas terapéuticas, específicamente enfocadas en el género y el papel de la familia en este tipo de patologías. Se observó que el género predominante es el femenino, al ser más vulnerable que el masculino $(4,6,8,9$, $23,24)$. Por otro lado, a lo largo de la discusión, se evidenció cómo una familia disfuncional no solo predispone a que el adolescente presente este trastorno sino que también ayuda a prolongarlo y empeorar su sintomatología. El conocimiento de estos hechos facilita el tratamiento del paciente pues evidencia el efecto que puede tener una terapia conjunta. Se espera que, con un análisis más profundo, se pueda poner en marcha un plan de prevención $(10,33)$ en los adolescentes teniendo en cuenta los factores que en ellos influyen sin dejar a un lado, por supuesto, a la familia que es indispensable en todo este proceso. 


\section{AGRADECIMIENTOS}

Al doctor Juan Carlos González, por su asesoría, apoyo y colaboración en el desarrollo de esta investigación. A la Fundación Universitaria Juan N. Corpas por brindarnos el espacio y, por último, al colegio IED de la localidad de Suba, por permitirnos realizar el trabajo de campo en sus instalaciones.

\section{REFERENCIAS}

(1) Martínez P, Zusman L, Hartley J, Morote R, Calderón A. Estudio epidemiológico de los trastornos alimentarios y factores asociados en Lima Metropolitana. [Internet]. Revista de Psicología de la PUCP [consultado el 29 de octubre de 2016]. 2003; XXI (2) Disponible en: https://dialnet.unirioja.es/descarga/articulo/994002.pdf

(2) Moreno P, Ochoa S, Vásquez D, Salazar D, Ortiz E, López Y et al. Trastornos de la conducta alimentaria en adolescentes de colegios públicos de Pereira. [Internet]. Rev. Méd. Risaralda [consultado 29 de octubre de 2016]. 2016; 22(1):9-13. Disponible en: http://biblioteca.universia.net/html_bura/ficha/params/title/trastornos-conducta-alimentariaadolescentes-colegios-publicos-pereira/id/67514903. html

(3) Gorag A, Iñarritu M. ¿Cómo ves? Trastornos de la conducta alimentaria. Anorexia y bulimia [Internet]. México: Dirección general de divulgación de la ciencia. 2005; [consultado: 23 de agosto de 2016]. Disponible en:

http://pregrado.juanncorpas.edu.co/pluginfile.php/27910/mod_book/chapter/66/2.\%20Guia\%20ElabCitas\%20y\%20Ref\%20Estilo\%20Vancouver.pdf

(4) Cruzat C, Ramírez P, Melipillán R, Marzolo P. Trastornos alimentarios y funcionamiento familiar percibido en una muestra de estudiantes secundarias de la Comuna de Concepción, Chile. Psykhe [Internet] [consultado el 29 de octubre de 2016]. 2008; 17(1): 81-90 Disponible en: http://www.redalyc.org/articulo.oa?id=96717108

(5) Méndez P, Vázquez V, García E. Los trastornos de la conducta alimentaria. México: Bol. Med. Hosp. Infant. Mex. [Internet]. 2008; 65(6): 579-592. [Consultado: 29 de octubre de 2016] Disponible en: http://www.scielo.org.mx/scielo.php?script=sci_arttext\&pid=S1665-11462008000600014

(6) Haller E. Eating Disorders a Review and Update. [Internet]. The Western Journal of Medicine [consultado el 5 de noviembre de 2016]. 1992. Disponible en:https://www.ncbi.nlm.nih.gov/pmc/articles/PMC1022101/pdf/westjmed00088-0054.pdf

(7) Ruíz M, Masjuan N, Costa-Ball D, Cracco C. Funcionamiento familiar y trastornos de la conducta alimentaria. [Internet]. Una investigación desde el modelo circumplejo. Ciencias Psicológicas [consultado 29 de octubre de 2016]. 2015; 9(2): 153-162. Disponible en: http://revistas.ucu.edu.uy/index.php/cienciaspsicologicas/article/view/439

8) Ruíz A, Vázquez R, Mancilla M, Viladrich C, Halley M. Factores familiares asociados a los trastornos alimentarios: una revisión [Internet]. Revista Mexicana de Trastornos Alimentarios [consultada 29 de octubre de 2016]. 2013; 4(1): 45-57. Disponible en:http://www.redalyc.org/articulo.0a?id=425741619006

(9) González, J. Restrepo, F. Relaciones entre los comportamientos de riesgo psicosociales y la familia en adolescentes de Suba, Bogotá. Rev. salud pública. [revista on-line] 2011 [consultado el 12 de noviembre de 2016]; 13 (1): 67-78. Disponible en: http://www.revistas.unal.edu.co/index.php/revsaludpublica/article/view/33530/38088

(10) Rutsztein G, Murawski B, Elizathe L, Arana F, Armatta A, Leonardelli E. Trastornos alimentarios en mujeres adolescentes: un estudio comparativo entre pacientes, estudiantes de danza y estudiantes de escuelas medias [Internet]. Revista Colombiana de Psiquiatría [consultado 29 de octubre de 2016]. 2019; 39(2): 329-346. Disponible en: danza y estudiantes de escuelas medias [Internet]. Revis

(11) Amaya A, Álvarez G, Mancilla JM. Insatisfacción corporal en interacción con autoestima, influencia de pares y dieta restrictiva: Una revisión. [Internet] Rev. Mex. de trastor aliment [consultado 29 de octubre de 2016]. 2010; 1(1): 76-89. Disponible en: http://www.scielo.org.mx/scielo.php?script=sci_arttext\&pid=S2007-15232010000100008

(12) Durán M, Morales M, Hernández M. La publicidad de televisión entre otros factores socioculturales influyentes en los trastornos de la conducta alimentaria. [Internet]. 2000 [consultado 29 de octubre de 2016] Disponible en: www.aeic2010malaga.org/upload/ok/47.pdf

(13) Moreno M, Ortiz G. Trastorno alimentario y su relación con la imagen corporal y la autoestima en adolescentes. Terapia psicológica [Internet]. Ter Psicol [consultado 29 de octubre de 2016]. 2009; 27(2): 181-190. Disponible en: http://www.scielo.cl/scielo.php?script=sci_arttext\&pid=S0718-48082009000200004

(14) Suárez M, Alcalá M. Apgar familiar: una herramienta para detectar disfunción familiar [Internet]. Rev. Méd. La Paz [consultado 12 de noviembre de 2016]. 2014; 20(1): 53-57. Disponible en: http://www.scielo.org.bo/scielo.php?script=sci_arttext\&pid=S172689582014000100010\&lng=es.

(15) García L. González E, López I. Ezquerra J. El test SCOFF: una posible herramienta de cribado de los trastornos de comportamiento alimentario en atención primaria. [Internet]. Aten Primaria [consultado el 19 de noviembre de 2016]. 2003; 32(5): 315-16. Disponible en: http://www.elsevier.es/es-revista-atencion-primaria-27-articulo-el-test-scoff-unaposible-13051586

(16) González JC. Riesgos y familia en la adolescencia. Un estudio acerca de los comportamientos de riesgos y la familia de los adolescentes de los colegios públicos. FUJNC. [Internet]. Nov 2007 [consultado el 12 de noviembre de 2016]. Disponible en: https://www.juanncorpas.edu.co/uploads/media/TEXTO RIESGOS FAMILIA Y A ADOLESCENTES 02.pdf

(17) González, J. Determinación de Relaciones entre los Comportamientos de Riesgo Psicosociales y la Familia de la Población Adolescente Escolar de los Colegios públicos de Suba. UNAL. [Internet] 2006 [citado el 12 de noviembre de 2016] Disponible en: http://www.bdigital.unal.edu.co/657/1/597353.2006.pdf

(18) Rueda G, Díaz L, Campo A, Barros JA, Ávila GC, Oróstegui LT et al. Validación de la Encuesta Scoff para tamizaje de trastornos de la conducta alimentaria en mujeres universitarias. Universidad Autónoma de Bucaramanga. [Internet]. Biomédica [consultado el 12 de noviembre de 2016]. 2005; 25: 196-202. Disponible en: http://www.revistabiomedica.org/index.php/biomedica/article/viewFile/1342/1457

(19) Gempeler J. Trastornos de la alimentación en hombres: cuatro subtipos clínicos. [Internet]. Revista Colombiana de Psiquiatría [consultado 15 de octubre de 2016]. 2006 XXXV(3): 352-361. Disponible en: http://www.redalyc.org/articulo. oa?id=80635305.

(20) Murawski B, Elizathe L, Rutsztein G. Hábitos alimentarios e insatisfacción con la imagen corporal. Un estudio comparativo entre mujeres y varones estudiantes de escuelas secundarias. [Internet]. Facultad de Psicología - UBA [citado 2016 Oct 15]. XVI: 65-72. Disponible en: http://www.scielo.org.ar/pdf/anuinv/v16/v16a06.pdf

(21) López P, Molina Z, Rojas, L. Influencia del género y la percepción de la imagen corporal en las conductas alimentarias de riesgo en adolescentes de Mérida. [Internet]. AnVenezNutr [consultado 6 de noviembre de 2016]. 2008; 21(2): 85-90. Disponible en: http://www.scielo.org.ve/scielo.php?script=sci_arttext\&pid=S0798$07522008000200005 \& \operatorname{lng}=\mathrm{es}$

(22) Galarsi MF, Correche MS, Ledezma C. Conductas y actitudes hacia la alimentación en una muestra de mujeres jóvenes. [Internet]. Fundamentos en Humanidades XI [consultado: 6 de noviembre de 2016]. 2010; XI(1): 193-205. Disponible en: http://www.redalyc.org/pdf/184/18415426013.pdf

(23) Maganto C, Cruz, S. La imagen corporal y los trastornos alimenticios: una cuestión de género. Facultad de Psicología. [Internet]. Universidad del País Vasco [consultado el 12 de Maganto C, Cruz, S. La imagen corporal y los trastornos alimenticios: una cuestión de género. Facultad de Psicología. [Inte

(24) López P, Prado J, Montilla M, Molina Z. Da Silva G, Arteaga F. Insatisfacción por la imagen corporal y la baja autoestima por la apariencia física en estudiantes de la facultad de Medicina de la Universidad de los Andes del Estado Mérida, Venezuela [Internet]. Revista MHSalud [consultado el 12 de noviembre de 2016]; 2008; 5(1). Disponible en: https://dialnet.unirioja.es/buscar/documentos?querysDismax.DOCUMENTAL_TODO=mujeres $+y+$ trastornos+alimenticios

(25) Perpiñá C, Baños R. Distorsión de la imagen corporal: Un estudio en adolescentes [Internet]. Anales de psicología [consultado el 12 de noviembre de 2016]. 1990; 6(1): 1-9. Disponible en: http://www.um.es/analesps/v06/v06_1/01-06_1.pdf

(26) Díaz J, Fernández M, Vázquez R, Álvarez G, Franco K, López X et al. Influencias socioculturales y conductas alimentarias no saludables en hombres y mujeres de España y México. [Internet]. Rev. Mex. detrastor. Aliment [consultado el 12 de noviembre de 2016]. 2010; 1(1). Disponible en: http://www.scielo.org.mx/scielo.php?script=sci_arttext\&pid=S2007-15232010000100005

(27) Toro J, Nieves K, Borrero N. Cuerpo y masculinidad: Los desórdenes alimentarios en hombres. [Internet]. InteramericanJournal of Psychology [consultado 6 de noviembre de 2016]. 2010; Pg 225-234. Disponible en: http://www.redalyc.org/articulo.oa?i d=28420641003

(28) Marmo, J. Parental styles and risk factors associated with eating pathology. Pontificia Universidad Católica. Av.psicol. [revista en la Internet] 22(2) 2014. [citado 2016 Oct 15] Disponible en: http://www.unife.edu.pe/publicaciones/revistas/psicologia/2014_2/165_Julieta_Marmo.pdf

(29) Sainos D, Sánchez M, Vázquez E, Gutiérrez I. Funcionalidad familiar en pacientes con anorexia nerviosa y bulimia. [Internet]. Atenfam [consultado el 12 de noviembre de 2016]. 2015; 22(2). Disponible en: http://www.revistas.unam.mx/index.php/atencion familiar/article/download/47995/43114

(30) Sim L, Homme J, Lteif A, Vande J, Schak K, Ellingson J. Family Functioning and Maternal Distress in Adolescent Girls with Anorexia Nervosa. [Internet]. Int J Eat Disord [consultado el 12 de noviembre de 2016]. 2009; 42:531-539. Disponible en: https://www.ncbi.nlm.nih.gov/pubmed/19189407

(31) González L, Hidalgo M, Hurtado M, Nova C Venegas M. Relación entre factores individuales y familiares de riesgo para desórdenes alimenticios en alumnos de enseñanza media. [Internet]. Revista de Psicología de la Universidad de Chile [consultado el 12 de octubre de 2016]. 2002; XI(1). Disponible en: http://www.redalyc.org/pdf/264/26411107.pdf

(32) Vazquez,R.Raich, R. El papel de la familia en los trastornos alimentarios. [Internet].Psicologia conductual [consultado el 23 de agosto de 2016]. 1997; 5(3): 391-407. Disponible en: http://www.funveca.org/revista/PDFespanol/1997/art05.3.05.pdf

(33) Rutsztein, G. Murawski, B. Elizathe, L. Scappatura, L. Trastornos alimentarios: Detección en adolescentes mujeres y varones de Buenos Aires. Un estudio de doble fase. [Internet]. Mexican Journal of Eating Disorders [consultado el 19 de noviembre de 2016]. 2010; 1: 48-61. Disponible en: http://journals.iztacala.unam.mx/index.php/amta/article/view/6

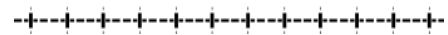

